\title{
Modificaciones Estructurales en el Estómago y el Hígado de Paralabrax maculatofasciatus (Steindacher, 1868) ante Situaciones de Estrés Crónico
}

\author{
Structural Modifications in the Stomach and Liver of Paralabrax maculatofasciatus \\ (Steindacher, 1868) under Chronic Stress Conditions
}

\author{
${ }^{*}$ M. Zacarias; ${ }^{* *}$ M. Cadena \& ${ }^{* * *} P$. Rivas
}

ZACARIAS, M.; CADENA, M. \& RIVAS, P. Modificaciones estructurales en el estómago y el hígado de Paralabrax maculatofasciatus (Steindacher, 1868) ante situaciones de estrés crónico. Int. J. Morphol., 27(2):425-433, 2009.

RESUMEN: Para determinar el efecto del estrés a nivel del aparato digestivo, organismos de la especie Paralabrax maculatofasciatus fueron sometidos a dos modelos de estrés: el primero por descenso en el nivel del agua (n=84) por 30 y 60 minutos diariamente, y el segundo por cultivo a altas densidades $(\mathrm{n}=96)$ de 1,6 y 3,2 kg/100 1. Se tomaron muestras de estómago e hígado y se fijaron en formol al 10\%. Los tejidos fueron incluidos en parafina, realizándose cortes de $6 \mu \mathrm{m}$ que se tiñeron con H-E. En ambas condiciones de estrés el estómago presentó cambios morfológicos importantes tales como hipertrofia y atrofia en la capa mucosa. En el hígado se observaron cambios de coloración y textura, así como hemólisis, inflamación y necrosis. Por descenso en el nivel del agua el estómago presentó diferencias morfométricas significativas $(\mathrm{P}<0,05)$, entre los diferentes tiempos de exposición al estrés y/o entre los días de muestreo en algunos de los parámetros medidos. Por cultivo a altas densidades, se observaron diferencias significativas en algunos parámetros, 4 de ellos presentaron interacción densidad-muestreo y el resto presentaron diferencias significativas sin interacción. El hígado presentó diferencias significativas $(\mathrm{P}<0,05)$ en el perímetro nuclear entre los diferentes días de muestreo. El estrés provocado por alta densidad de cultivo resultó ser el factor que provocó daños tisulares más severos en los órganos estudiados, de ahí la importancia de mantener las condiciones adecuadas en los cultivos, como son el nivel de agua y el número de organismos por estanque.

PALABRAS CLAVE: Estrés; Paralabrax maculatofasciatus; Histopatología.

\section{INTRODUCCIÓN}

En todas las piscifactorias se pueden llegar a presentar condiciones desfavorables que someten a los organismos a una situación de estrés permanente y en aumento, reduciendo significativamente la calidad de vida de los peces en cultivo (Jiménez et al., 1986). El estrés es considerado como un factor determinante e importante en la aparición, establecimiento y propagación de enfermedades (Sindermann, 1977; Hästein \& Lindstad, 1991; Pickering, 1993). Diversos signos pueden detectarse a simple vista en los peces que son sometidos a estrés, entre los más frecuentes se encuentran: alteraciones en la función cardíaca (Titu, 2002), descamación, producción de moco (Hernández, 2006), mortalidad en masa, pereza, hidropesía, decoloración de la piel, hemorragias en branquias, exoftalmia, etc. (Roberts, 1975; Jiménez et al.).
Para medir el efecto del estrés, los trabajos que se utilizan con más frecuencia se basan en trastornos fisiológicos tales como modificaciones en las concentraciones de corticosteroides y glucosa en plasma (Mazeaud et al., 1977; Donaldson, 1981; Tave, 1994; Walling \& Van Dén Avyle, 1995). Otra forma de valorar el estrés es analizar el nivel tisular, en órganos blanco que se ven alterados estructuralmente como respuesta a estímulos estresantes. De menor a mayor grado se pueden observar procesos inflamatorios, agudos o crónicos a nivel celular y tisular; degeneración, procesos de reparación y regeneración, neoplasias y finalmente trastornos genéticos (Sindermann, 1990; Anderson, 1990). La realización de estudios destinados a conocer las diversas respuestas de los organismos frente al estrés resulta de suma importancia, sobre todo económica,

\footnotetext{
* Laboratorio Experimental de Maricultura, Unidad Pichilingue. Universidad Autónoma de Baja California Sur. México.

** Laboratorio Experimental de Maricultura, Unidad Pichilingue. Universidad Autónoma de Baja California Sur. México.

*** Laboratorio de Biología de la Reproducción Animal, Facultad de Ciencias. Universidad Nacional Autónoma de México. México.
} 
ya que por efecto del estrés se pueden llegar a mermar en gran medida las poblaciones en cultivo. Las alteraciones histopatológicas en los tejidos animales, reflejan cambios bioquímicos y fisiológicos adversos, por lo que son poderosos indicadores de exposición a estresantes ambientales (Hinton \& Laurén, 1990). El análisis morfométrico de estructuras tisulares nos permite realizar el análisis cuantitativo de las alteraciones provocadas por el estrés y junto con la histopatología, permiten evaluar las condiciones de salud de las poblaciones en cultivo (Goede \& Barton, 1990).

En este trabajo se estimó, a nivel tisular, la patología del estómago y del hígado en individuos sometidos a estrés, comparándose morfométricamente algunos parámetros tisulares de ambos órganos con sus controles.

\section{MATERIAL Y MÉTODO}

Modelo de estrés 1: Descenso en el nivel del agua. Ochenta y cuatros individuos de la especie $P$. maculatofasciatus fueron colocados en estanques circulares de fibra de vidrio con fondo plano y 2001 de capacidad, utilizándose un sistema de recirculación semicerrado y alimentándose a saciedad una vez al día. Se utilizaron dos lotes experimentales ( $n=14$ cada uno) a los cuales se les descendió el nivel del agua por 30 minutos (lote 1) y 60 minutos (lote 2), y un lote control $(n=14)$ al cual no se le modificó el nivel del agua. Cada lote contó con dos réplicas. El descenso en el nivel del agua se realizó diariamente en los lotes experimentales a las 10:00 A.M., manteniendo una capa de agua lo suficientemente alta para cubrir el dorso de los peces. Después de 30 o 60 minutos respectivamente, se reestableció el nivel del agua a la condición normal. Se realizaron tres muestreos en cada grupo experimental, uno al inicio del experimento, el segundo a los 30 días y el último a los 60 días. En cada muestreo se extrajeron 4 organismos por condición experimental y por réplica. Los peces fueron anestesiados profundamente por hipotermia durante $20 \mathrm{~min}$ y posteriormente se cortó el cordón espinal (Olfert et al., 1998). Se tomaron muestras de 1 $\mathrm{cm}^{3}$ de estómago e hígado y se fijaron en formol al 10\%. Las muestras fueron sometidas a los procesos de deshidratación e inclusión en parafina. Se realizaron cortes de $6 \mu \mathrm{m}$ de espesor y se tiñeron con la técnica de H-E (Estrada et al., 1982).

Modelo de estrés 2: Cultivo a altas densidades. Se utilizaron dos densidades experimentales, $1,6 \mathrm{~kg} / 1001$ y $3,2 \mathrm{~kg} /$ 1001 , y el control con $0,58 \mathrm{~kg} / 100 \mathrm{l}$, empleándose un total de 96 organismos. Cada densidad contó con 3 grupos. El primer grupo se utilizó al inicio de la experimentación, el segundo a los 30 días y el último a los 60 días. En cada muestreo se extrajeron 4 organismos por condición experi- mental y se sacrificaron por hipotermia y corte del cordón espinal. Se tomaron muestras de estómago e hígado, las cuales se procesaron como se mencionó anteriormente.

Análisis morfométrico. Para la revisión e interpretación histológica se utilizó un microscopio Spencer de campo claro. En el estómago se analizaron 5 campos equivalentes por individuo $(\mathrm{n}=12$ por condición experimental incluyendo a los controles y por muestreo), en cortes transversales de la capa mucosa de la región fúndica. La altura del epitelio de revestimiento, la longitud de la cripta mucosa y de la glándula gástrica, así como el diámetro mayor y menor de las células mucosas y gástricas se midieron utilizando una reglilla ocular. La altura de las glándulas gástricas se midió a 10X, en tanto que la longitud de las glándulas mucosas y la altura del epitelio fueron medidos a 40X. Los diámetros celulares fueron medidos a 100X, y la densidad celular fue determinada contando el número de células mucosas o células glandulares gástricas en $10 \mathrm{~mm}^{2}$, usando una cuadrícula ocular a 10X.

En el hígado se seleccionaron al azar 5 campos, midiéndose el área y perímetro nuclear de 5 hepatocitos por campo, por individuo a 200X $(\mathrm{n}=12$ por condición experimental incluyendo a los controles y por muestreo). Al localizarse zonas de necrosis se procedió a medir el porcentaje de tejido necrosado con respecto del tejido sano (área $=9,5$ $\mathrm{mm}^{2}$ ), muestreándose 20 campos diferentes a un aumento de 32X. Todos estos datos fueron obtenidos por digitalización de las imágenes utilizando el programa IPPLUS 2.0. Las medidas morfométricas utilizadas en cada uno de los órganos fueron las mismas para ambas pruebas de aplicación de estrés y sus controles.

Análisis de datos. Se realizó un análisis de varianza de dos vías con interacción entre factores y la prueba de Tukey para cada variable de respuesta, utilizándose el paquete estadístico SAS (versión 1988).

\section{RESULTADOS}

Efecto del estrés por descenso en el nivel del agua. En el estómago macroscópicamente no se observaron cambios, no así a nivel microscópico en donde se detectaron cambios en el tamaño de las células gástricas y mucosas, comparadas con el control (Fig. 1a). En el análisis morfométrico se obtuvo una disminución significativas en la longitud de las glándulas gástricas y un incremento significativo en el diámetro mayor de las células mucosas entre los 0 y 30 min de exposición al estrés, y en el diámetro menor de las células gástricas entre los 30 y 60 min (Fig. 1b) (Tabla I). 
Tabla I. Media \pm DE $(\mu \mathrm{m})$ de las diferentes variables del estómago de $P$. maculatofasciatus, sometida a estrés por descenso en el nivel del agua, entre los diferentes tiempos de exposición al estrés ( 0,30 y 60 minutos).

\begin{tabular}{llccc}
\hline \multicolumn{1}{c}{ VARIABLE } & N & Control & 30 minutos & 60 minutos \\
\hline Altura del epitelio & 36 & $35,50 \pm 6,57$ & $39,542 \pm 5.89$ & $38,295 \pm 12,21$ \\
Longitud de la cripta mucosa & 36 & $77,07 \pm 18,27$ & $79,83 \pm 13.21$ & $84,22 \pm 40,42$ \\
Longitud de la glándula & 36 & $309,52 \pm 36,5^{\mathrm{a}}$ & $270,14 \pm 48.23^{\mathrm{b}}$ & $283,43 \pm 33,26^{\mathrm{ab}}$ \\
Diámetro menor de la célula gástrica & 36 & $9,909 \pm, 82^{\mathrm{ab}}$ & $9,363 \pm 1.29^{\mathrm{a}}$ & $10,458 \pm 1,45^{\mathrm{b}}$ \\
Diámetro mayor de la célula gástrica & 36 & $26,136 \pm 3,37$ & $26,728 \pm 3.76$ & $26,858 \pm 2,34$ \\
Diámetro menor de la célula mucosa & 36 & $8,774 \pm, 56$ & $9,509 \pm 1.03$ & $8,892 \pm, 64$ \\
Diámetro mayor de la célula mucosa & 36 & $17,044 \pm 1,55^{\mathrm{a}}$ & $18,916 \pm 1.89^{\mathrm{b}}$ & $17,858 \pm 2,18^{\mathrm{ab}}$ \\
Número de células gástricas & 36 & $35,444 \pm 4,39$ & $36,333 \pm 3.1$ & $38,083 \pm 3,42$ \\
Número de células mucosas & 36 & $2, .250 \pm 3,5$ & $26,500 \pm 4.18$ & $26,833 \pm 3,05$ \\
\hline
\end{tabular}

a,b Variables que presentaron diferencias significativas $(\mathrm{P}<0.05)$.
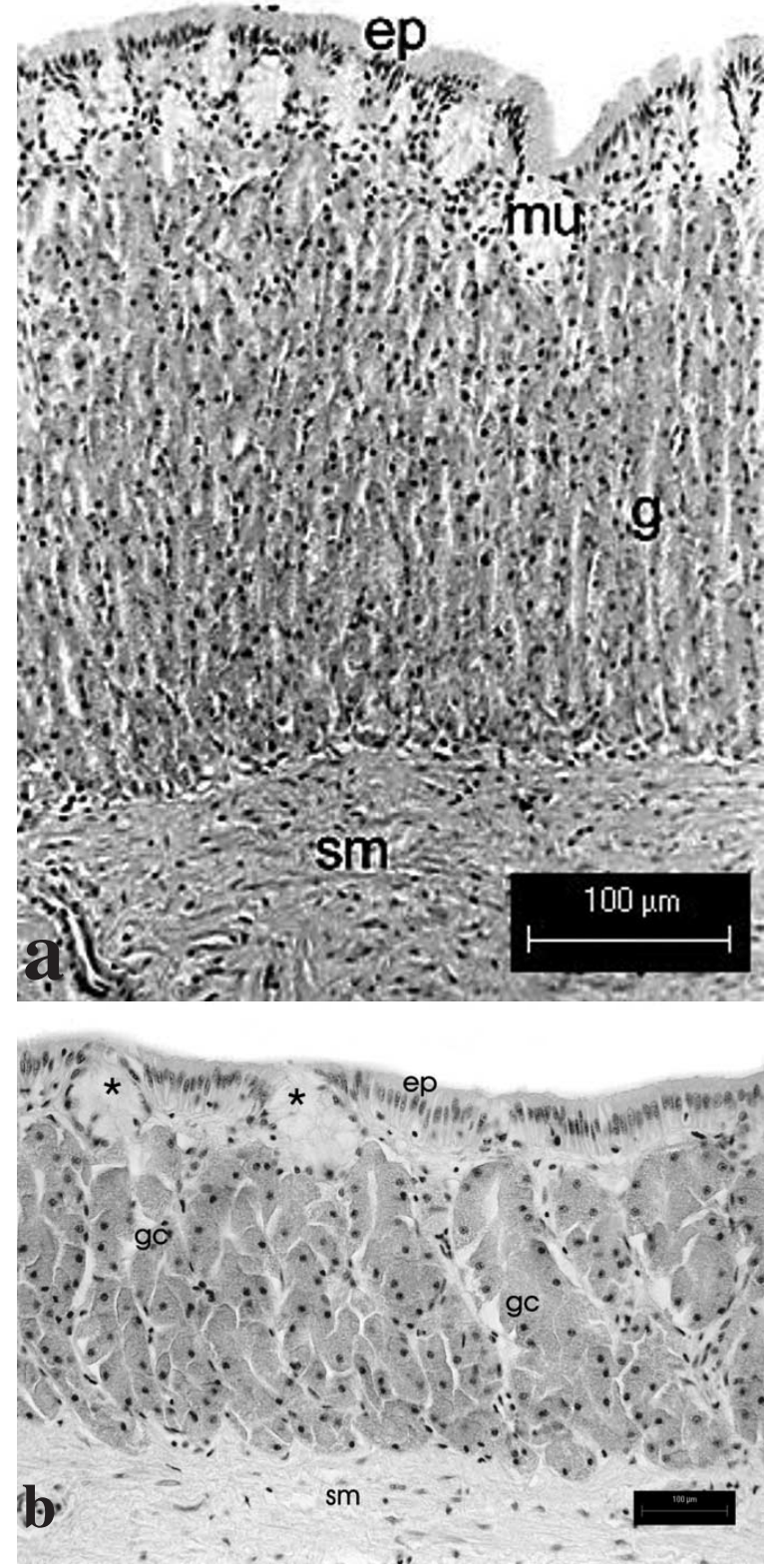

Entre los 0,30 y 60 días se observaron incrementos significativos $(\mathrm{P}<0,05)$ en la longitud de las glándulas gástricas, el diámetro mayor de las células gástricas y el diámetro mayor de las células mucosas (Fig. 1c); todas estas diferencias se observaron entre el muestreo inicial y el dia $30 \mathrm{y}$ el muestreo inicial y el día 60, no encontrándose diferencias entre los 30 y 60 días. La única variable con diferencias significativas entre los tres muestreos, fué la disminución del diámetro menor de las células gástricas. Por otro lado, la densidad de las células mucosas incrementó solo al final del experimento (Tabla II).

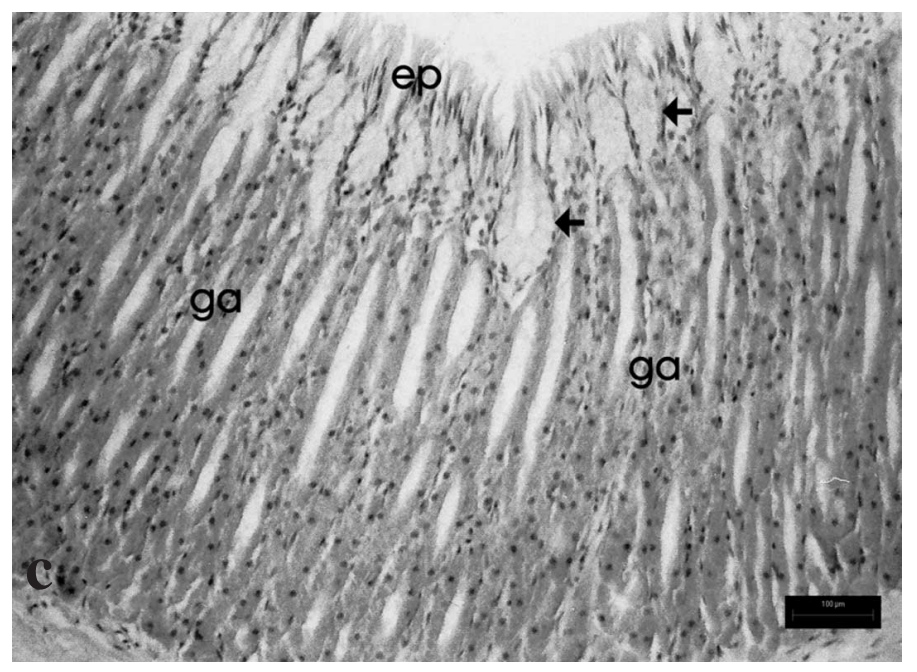

Fig. 1. Secciones longitudinales del estómago de P. maculatofasciatus en organismos sometidos a estrés por descenso en el nivel del agua. a) organismo control; epitelio cilíndrico simple (ep), criptas mucosas (mu), glándulas gástricas (g), submucosa ( $\mathrm{sm})$; b) corte longitudinal a nivel de la mucosa en organismos con 30 min de exposición al estrés; epitelio cilíndrico simple (ep), células mucosas (*), glándulas gástricas cortas con células hipertróficas (gc), submucosa ( $\mathrm{sm})$; c) corte longitudinal a nivel de la mucosa a los 60 días de exposición al estrés; epitelio cilíndrico simple (ep), hiperplasia de glándulas mucosas $(\rightarrow)$, glándulas gástricas adelgazadas con células atróficas (ga). H-E 

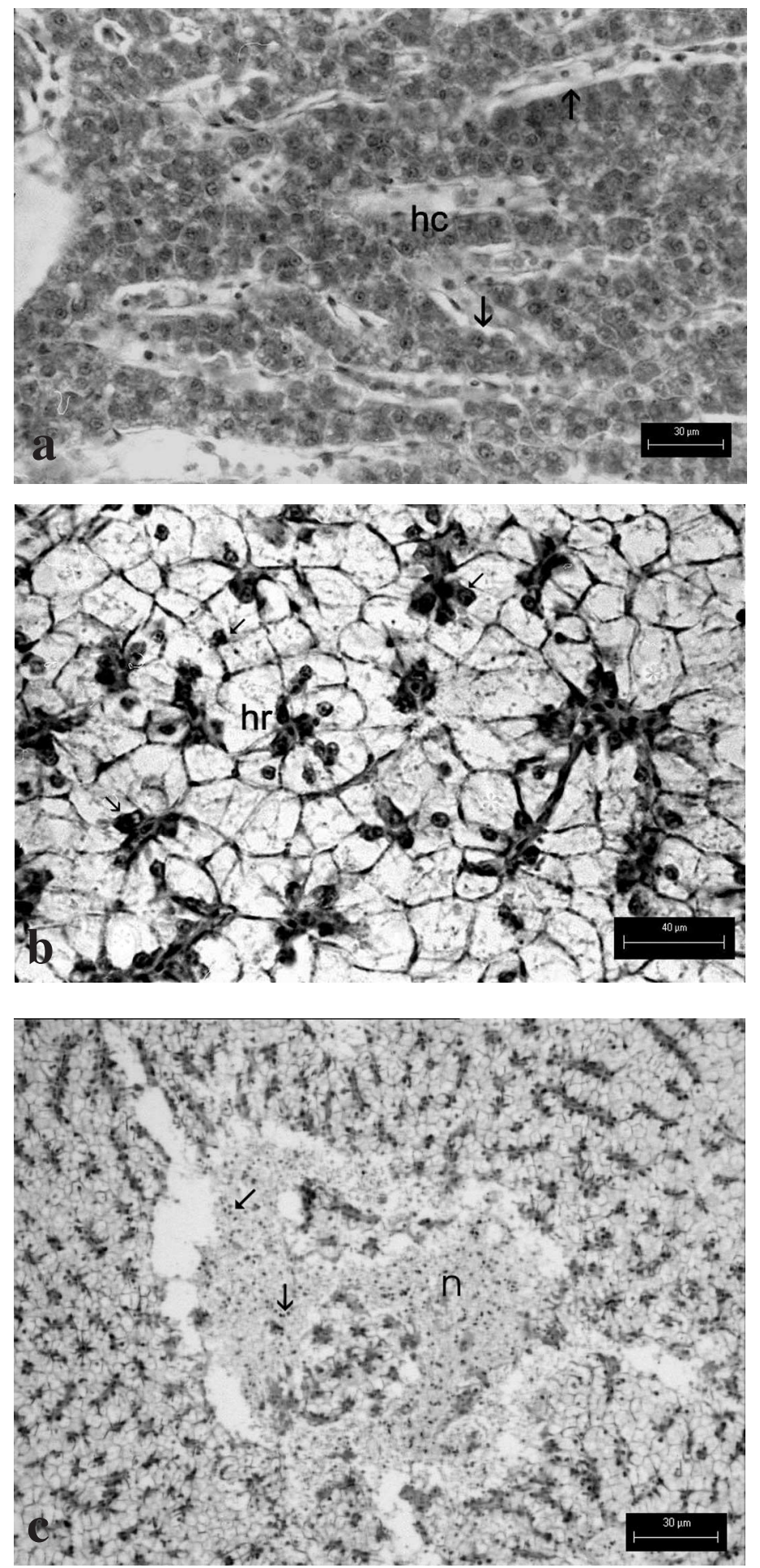

Fig. 2. Secciones transversales del hígado de $P$. maculatofasciatus en organismos sometidos a estrés por descenso en el nivel del agua; a) organismo control mostrando a los hepatocitos arreglados en cordones (hc), sinusoides $(\rightarrow)$; b) en organismos con 30 minutos de exposición al estrés los hepatocitos formaron rosetas (hr) y presentaron picnosis nuclear $(\rightarrow)$; c) a los 60 días de estrés se observaron áreas necróticas (n) e infiltrados celulares inflamatorios $(\rightarrow)$. H-E.
El hígado presentó un notable cambio de coloración, pasando de rosa pálido a amarillo-grisáceo, mostrándose friable al tacto. A nivel microscópico, el control (Fig. 2a) mostró a los hepatocitos arreglados en cordones y rodeando a los sinusoides; en los organismos sometidos al descenso del agua diariamente por 60 minutos se observó disminución del tamaño de los hepatocitos; a los 30 y 60 minutos se modificó el arreglo de los cordones hepáticos adoptando un arreglo en forma de roseta (Fig. 2b), y se observaron algunas zonas de necrosis, las cuales alcanzaron hasta un 2,5\% de tejido necrosado a los 60 días de estrés (Fig. 2c). En estas zonas se observaron hepatocitos en degeneración, picnosis nuclear, infiltrados celulares inflamatorios y hepatitis leve multifocal, comparada con el control. No se encontraron diferencias significativas en el área y perímetro de los núcleos de los hepatocitos en los tiempos de exposición al estrés $(0$, 30 y $60 \mathrm{~min})$, ni entre los dias de muestreo $(0,30$ y 60 días).

Efecto del estrés por cultivo a altas densidades. A simple vista el estómago presentó un ligero adelgazamiento en sus paredes. A nivel microscópico se observó aumento en la longitud de las criptas mucosas y de las glándulas gástricas (Fig. 3a). También se observaron campos con atrofia, hiperplasia ó metaplasia en las glándulas gástricas y en las criptas mucosas (Fig. 3b), comparadas con el grupo control (Fig. 1a).

El análisis morfométrico reveló diferencias significativas $(\mathrm{P}<0,05)$ con interacción DensidadMuestreo con disminución en el diámetro menor de las células de las glándulas gástricas y aumento en el número de células mucosas y gástricas. Entre las densidades de cultivo algunas diferencias fueron detectadas en la longitud de las criptas mucosas, con aumento entre el control y la densidad experimental $1,6 \mathrm{Kg}$ / 100 1. El diámetro menor de las células mucosas mostró disminución respecto al control en la densidad de 3,2 Kg/100 1 (Tabla III).

Así mismo, se encontraron aumentos entre los días de muestreo (0, 30 y 60 días), en la longitud de las criptas mucosas, la longitud de las glándulas gástricas y el diámetro mayor de sus células, obteniéndose diferencias significativas entre el primero y segundo muestreo y entre el primero y el tercero. Otras diferencias fueron obtenidas en ambos diámetros de las células mucosas, pero en este caso solo se observó entre el muestreo inicial y el final. Además, se encontraron aumentos significativos $(\mathrm{P}<0,05)$ con interacción densidad-muestreo en la densidad de las células gástricas y mucosas (Tabla IV). 
Tabla II. Media \pm DE $(\mu \mathrm{m})$ de las diferentes variables del estómago de P. maculatofasciatus, sometida a estrés por descenso en el nivel del agua, a los 0,30 y 60 días de estrés.

\begin{tabular}{llccc}
\hline \multicolumn{1}{c}{ Variable } & $\mathrm{N}$ & 0 días & 30 días & 60 días \\
\hline Altura del epitelio & 36 & $41,917 \pm 11,72$ & $36,833 \pm 5,32$ & $34,587 \pm 6,3$ \\
Longitud de la cripta mucosa & 36 & $75,84 \pm 18,06$ & $73,37 \pm 13,02$ & $91,90 \pm 31,19$ \\
Longitud de la glándula & 36 & $256,76 \pm 51,83^{\mathrm{a}}$ & $304,77 \pm 17,92^{\mathrm{b}}$ & $301,56 \pm 32,57^{\mathrm{b}}$ \\
Diámetro menor de la célula gástrica & 36 & $10,947 \pm 1,18^{\mathrm{a}}$ & $9,900 \pm, 7^{\mathrm{b}}$ & $8,883 \pm, 93^{\mathrm{c}}$ \\
Diámetro mayor de la célula gástrica & 36 & $23,520 \pm 2,92^{\mathrm{a}}$ & $28,302 \pm 1,4^{\mathrm{b}}$ & $27,900 \pm 2,33^{\mathrm{b}}$ \\
Diámetro menor de la célula mucosa & 36 & $8,807 \pm, 89$ & $9,451 \pm, 79$ & $8,917 \pm, 68$ \\
Diámetro mayor de la célula mucosa & 36 & $16,144 \pm 1,5^{\mathrm{a}}$ & $18,458 \pm 1,43^{\mathrm{b}}$ & $19,217 \pm 1,65^{\mathrm{b}}$ \\
Número de células gástricas & 36 & $35,583 \pm 3,8$ & $37,917 \pm 4,39$ & $36,361 \pm 2,79$ \\
Número de células mucosas & 36 & $25,278 \pm 3,01^{\mathrm{a}}$ & $25,167 \pm 3,06^{\mathrm{a}}$ & $29,139 \pm 3,09^{\mathrm{b}}$ \\
\hline
\end{tabular}

a, b, $c$ Variables que presentaron diferencia significativa $(\mathrm{P}<0.05)$.

Tabla III Media \pm DE $(\mu \mathrm{m})$ de las diferentes variables del estómago de P. maculatofasciatus, sometida a estrés por cultivo a altas densidades (Control = $0.58 \mathrm{Kg} / 100$ l, Densidad $1=1.6 \mathrm{Kg} / 100$ l, Densidad $2=3.2 \mathrm{Kg} / 100$ l).

\begin{tabular}{llccc}
\hline \multicolumn{1}{c}{ Variable } & $\mathrm{N}$ & Control & Densidad 1 & Densidad 2 \\
\hline Altura del epitelio & 36 & $35,500 \pm 6,57$ & $40,000 \pm 5,54$ & $42,708 \pm 11,26$ \\
Longitud de la cripta mucosa & 36 & $77,072 \pm 18,36^{\mathrm{a}}$ & $97,625 \pm 21,43^{\mathrm{b}}$ & $88,642 \pm 19,65^{\mathrm{ab}}$ \\
Longitud de la glándula & 36 & $309,52 \pm 36,5$ & $279,46 \pm 62,13$ & $288,28 \pm 44,09$ \\
Diámetro menor de la célula gástrica & 36 & $9,909 \pm, 82^{\mathrm{a}}$ & $7,904 \pm 2,04^{\mathrm{b}}$ & $8,550 \pm 2,53^{\mathrm{a}}$ \\
Diámetro mayor de la célula gástrica & 36 & $26,136 \pm 3,37$ & $24,851 \pm 2,8$ & $25,767 \pm 2,02$ \\
Diámetro menor de la célula mucosa & 36 & $8,774 \pm, 56^{\mathrm{a}}$ & $8,034 \pm 1,36^{\mathrm{ab}}$ & $7,625 \pm, 82^{\mathrm{b}}$ \\
Diámetro mayor de la célula mucosa & 36 & $17,044 \pm 1,54$ & $18,374 \pm 2,27$ & $17,076 \pm 2,19$ \\
Número de células gástricas * & 36 & $35,444 \pm 4,39^{\mathrm{a}}$ & $44,917 \pm 8,4^{\mathrm{b}}$ & $45,889 \pm 7,6^{\mathrm{b}}$ \\
Número de células mucosas * & 36 & $26,250 \pm 3,5^{\mathrm{a}}$ & $31,361 \pm 6,02^{\mathrm{b}}$ & $30,917 \pm 6,36^{\mathrm{b}}$ \\
\hline
\end{tabular}

a, b Variables que presentaron diferencias significativas $(\mathrm{p}<0.05)$

* Variables que presentaron interacción Densidad-Muestreo $(\mathrm{p}<0.05)$.

Tabla IV. Media \pm DE $(\mu \mathrm{m})$ de las diferentes variables del estómago de $P$. maculatofasciatus, sometida a estrés por cultivo a altas densidades a los 0,30 y 60 días de cultivo.

\begin{tabular}{lcccc}
\hline \multicolumn{1}{c}{ Variable } & $\mathrm{N}$ & 0 días & 30 días & 60 días \\
\hline Altura del epitelio & 36 & $41,917 \pm 11,72$ & $38,417 \pm 5,12$ & $37,875 \pm 7,44$ \\
Longitud de la cripta mucosa & 36 & $75,842 \pm 18,06^{\mathrm{a}}$ & $92,350 \pm 22,24^{\mathrm{b}}$ & $95,147 \pm 18,86^{\mathrm{b}}$ \\
Longitud de la glándula & 36 & $256,76 \pm 51,83^{\mathrm{a}}$ & $307,56 \pm 28^{\mathrm{b}}$ & $312,95 \pm 45,72^{\mathrm{b}}$ \\
Diámetro menor de la célula gástrica & 36 & $10,947 \pm 1,18^{\mathrm{a}}$ & $7,700 \pm 1,66^{\mathrm{b}}$ & $7,717 \pm 1,33^{\mathrm{b}}$ \\
Diámetro mayor de la célula gástrica & 36 & $23,520 \pm 2,92^{\mathrm{a}}$ & $26,383 \pm 2,1^{\mathrm{b}}$ & $26,850 \pm 2,05^{\mathrm{b}}$ \\
Diámetro menor de la célula mucosa & 36 & $8,807 \pm, 89^{\mathrm{a}}$ & $7,959 \pm 1,0^{\mathrm{ab}}$ & $7,667 \pm 1,01^{\mathrm{b}}$ \\
Diámetro mayor de la célula mucosa & 36 & $16,144 \pm 1,5^{\mathrm{a}}$ & $17,510 \pm 2,06^{\mathrm{ab}}$ & $18,842 \pm 1,78^{\mathrm{b}}$ \\
Número de células gástricas * & 36 & $35,583 \pm 3,8^{\mathrm{a}}$ & $45,667 \pm 9,21^{\mathrm{b}}$ & $45,000 \pm 7,2^{\mathrm{b}}$ \\
Número de células mucosas * & 36 & $25,278 \pm 3,01$ & $30,167 \pm 6,39$ & $33,083 \pm 4,75$ \\
\hline
\end{tabular}

${ }^{\mathrm{a}, \mathrm{b}}$ Variables que presentaron diferencias significativas $(\mathrm{p}<0.05)$.

*Variables que presentaron interacción Densidad-Muestreo $(\mathrm{p}<0.05)$.

Como en el caso de estrés por el descenso en el nivel del agua, el hígado mostró un cambio evidente de color de rosa pálido a amarillo-grisáceo y cambio de textura, volviéndose friable al tacto. A nivel microscópico se observó picnosis nuclear, hemólisis, colangio hepatitis multifocal, infiltrados celulares inflamatorios y necrosis severa, alcanzando hasta el 26,2\% de tejido necrótico en la densidad de 1,6 $\mathrm{Kg} / 100$ 1, y el 20\% de tejido necrótico en los organismos 
cultivados en la densidad de 3,2 Kg/100 1. El análisis morfométrico reveló la existencia de decrementos significativos $(\mathrm{P}<0,05)$ en el perímetro nuclear entre los días de

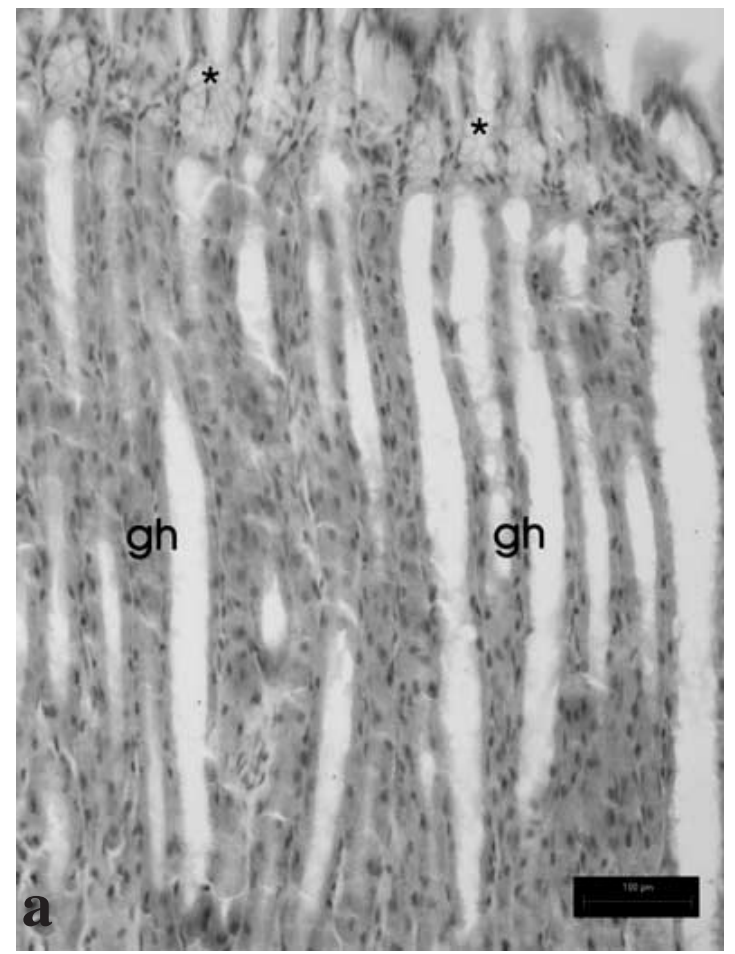

muestreo. Al aplicar la pruba de Tukey se observó que el muestreo inicial fue el que difirió significativamente de los otros dos (Tabla V).

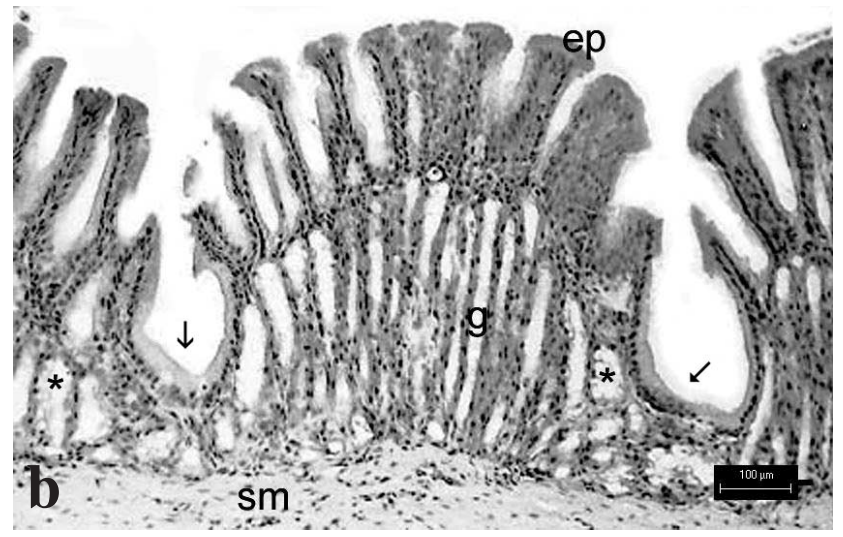

Fig. 3. Secciones longitudinales del estómago de $P$. maculatofasciatus sometidos a estrés por cultivo a altas densidades. a) corte longitudinal a nivel de la mucosa a los 60 días de cultivo a altas densidades, criptas mucosas hipertróficas (*) y glándulas gástricas hipeplásicas (gh); b) corte longitudinal a nivel de la mucosa, criptas mucosas metaplásicas $(\rightarrow)$ y atróficas $(*)$, glándulas gástricas atróficas (g), epitelio cilíndrico simple (ep), submucosa (sm). H. E.

Tabla V. Media \pm DE $(\mu \mathrm{m})$ de las diferentes variables del hígado de $P$. maculatofasciatus, sometida a estrés por cultivo a altas densidades, durante los diferentes muestreos (0, 30 y 60 días).

\begin{tabular}{ccccc}
\hline Variable & N & Control & 30 días & 60 días \\
\hline Área del núcleo & 36 & $26,30 \pm 2,91$ & $23,96 \pm 3.62$ & $24,46 \pm 3,04$ \\
Perímetro nuclear $^{\mathrm{a}}$ & 36 & $22,75 \pm 1,19^{\mathrm{a}}$ & $20,48 \pm 1.82^{\mathrm{b}}$ & $20,70 \pm 1,37^{\mathrm{b}}$ \\
\hline
\end{tabular}

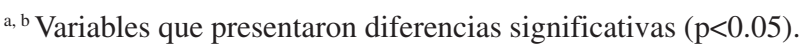

\section{DISCUSIÓN}

Los resultados de los análisis morfológico y morfométrico muestran que el mayor daño producido por el estrés a nivel tisular se localizó en el estómago, produciéndose numerosas alteraciones principalmente a nivel de la capa mucosa, esto debido a que, como menciona Ferguson (1992), la mucosa desempeña un papel fundamental como zona de protección, siendo la primera barrera de defensa de este órgano. Dentro de esta capa, las criptas mucosas y las glándulas gástricas, así como los diferentes tipos celulares que las componen, fueron los que mostraron mayores cambios.

Diversos han sido los trabajos en donde ubican al estómago como un órgano blanco indicador de la exposi- ción a factores estresantes. Peters et al. (1980) reportan que el estrés producido por dominancia social, por exposición al descenso en el nivel del agua y alta densidad de cultivo en anguilas, provocan variaciones en el diámetro del estómago, así como adelgazamiento de la mucosa gástrica, acompañados por erosión y descamación. Cousin et al. (1986), observaron regresión del tracto digestivo y diferentes grados de descamación en la mucosa digestiva en larvas de Turbot (Scophthalmus maximus L.), cultivadas a altas densidades.

En este trabajo se observó incremento en el número de células secretoras de moco y diversas transformaciones en las poblaciones celulares gástricas como hipertrofia, adel- 
gazamiento o atrofia celular tanto en las células gástricas como en las mucosas, las cuales son comunes en los peces y se consideran como un mecanismo de defensa contra agentes internos o externos (Jiménez et al.). Lesel (1980) y Sinderman (1990) han reportado que el estrés provoca una transformación a nivel de la mucosa aumentando el número de células secretoras de moco en cuya secreción se encuentran gran cantidad de anticuerpos, los cuales tienen como función principal participar en la defensa contra una posible invasión microbiana.

En el hígado, el cambio de coloración y de textura en comparación con los organismos control, fueron características generalizadas para todos los individuos, independientemente de la condición estresante a la que fueron sometidos. Goede \& Barton también observaron estos cambios en coloración y consistencia en el hígado, en peces sometidos a estrés. Solo en los organismos sometidos a estrés por alta densidad de cultivo, el perímetro nuclear de los hepatocitos disminuyó, tornándose irregular, presentando diferencias significativas entre el muestreo inicial y los dos restantes. Esta característica de cambio de forma del núcleo, como lo mencionan Hinton \& Laurén, es un marcador característico indicativo de estrés en los peces y que a su vez puede presentarse acompañado, como en el caso de este trabajo, de muerte celular, zonas necróticas, picnosis nuclear y cariólisis. Cahn (1975); Establier et al. (1978); Peters et al.; Wolf \& Smith (1981); Wedemeyer \& McLeay (1981); Brown et al. (1984); Fjolstad \& Heyeraas (1985) y Hernández (2006), también han encontrado cambios debidos al estrés tales como agotamiento de glucógeno en los hepatocitos, linfopoyesis, tumefacciones, gran vascularización, desorganización del parénquima, aumento en el grosor de los capilares, aumento en la basofilia nuclear y citoplasmática, infiltraciones de leucocitos y macrófagos, hemorragias, dilatación, hipertrofia e hiperplasia de los sinusoides, inflamación, núcleos picnóticos, presencia de pequeñas masas musculares, hiperplasia del tejido conjuntivo que rodea al conducto biliar, cariorexis, cariomegalia, neoplasias, carcinomas e infiltraciones de fibroblastos. La exposición a diversos insecticidas y organoclorados también pueden provocar muchas alteraciones en el hígado, como hipertorofia, inflamación, desorganización del parénquima, necrosis y vacuolización en el citoplasma (Salam et al., 2001; Olojo et al., 2005).

Nuestros resultados mostraron reorganización del parénquima, hepatocitos formando rosetas, infiltrados de leucocitos y macrófagos en relación directa con las áreas necróticas, núcleos picnóticos, congestión e hipertrofia del tejido conjuntivo de la periferia de los conductos biliares. Todas estas modificaciones nos indican que el hígado funciona como uno de los principales órganos blanco que res- ponde al estrés, lo cual Cahn justifica, afirmando que el hígado es un órgano que puede ser altamente dañado, dado su papel destoxificante y su participación activa en el metabolismo general del organismo, el cual se ve intensamente modificado por el estrés.

En este trabajo no se observaron cambios en el comportamiento, nado, color, apetito, etc. de los animales en experimentación. Sin embargo, se han reportado cambios en el comportamiento, en respuesta al estrés por cambios en la temperatura, como son el incremento en actividad, espasmos musculares, nado vertical y pérdida del equilibrio (Hernández, 1998; Del Rio, 2004). Afecciones como las encontradas aquí, pudieran ser interpretadas como algunas de las causas de las muertes aisladas o en masa, que se han reportado como decesos de origen desconocido, ya que no se han encontrado agentes virales o bacterianos, deficiencias alimenticias, fuentes de contaminación, etc., que las hubieran provocado (Jiménez et al.).

A nivel tisular, Goede \& Barton, así como Hinton \& Laurén, proponen que los cambios histopatológicos son indicadores de exposición a estresantes ambientales, y que estos cambios se dan como resultado de alteraciones bioquímicas y fisiológicas en los organismos, por lo que el tejido se transformará para tratar de mantener su homeostasis. Si el estrés es continuo provocará cambios grotescos en las estructuras de los tejidos u órganos. Estos efectos se apreciarán como un espectro de cambios necróticos en el estómago y el hígado, destacando la primera indicación de citotoxicidad en los individuos, como lo observado en este estudio.

Como pudimos observar, situaciones de estrés tales como descenso en el nivel del agua en los estanques y el mantenimiento de los individuos en densidades no apropiadas, afectan de manera directa a órganos blanco como son el estómago y el hígado; todo esto a la larga, afecta la calidad de vida de los animales disminuyendo su capacidad para tolerar otro factor estresante adicional.

Por todo esto se vislumbra que los estudios a nivel tisular de los efectos del estrés en organismos en cultivo resultan de suma importancia, ya que la información que se obtenga de éstos, será de gran ayuda para verificar el estado de salud de las poblaciones en cultivo y se evidenciará si de alguna manera, la exposición a un estresante ambiental está provocando efectos negativos en la sobrevivencia de los peces.

\section{AGRADECIMIENTOS}

Agradecemos a Barbosa, M. y a Benitez, J. por su valiosa asistencia técnica. 
ZACARIAS, M.; CADENA, M. \& RIVAS, P. Structural modifications in the stomach and liver of Paralabrax maculatofasciatus (Steindacher, 1868) under chronic stress conditions. Int. J. Morphol., 27(2):425-433, 2009.

SUMMARY: To determine stress effects at histological level in the digestive system, organisms of Paralabrax maculatofasciatus were submitted to 2 stress models. One of decreasing the water level $(n=84)$ for 30 and 60 min daily, and another at high density cultures $(\mathrm{n}=96)$ of 1.6 and $3.2 \mathrm{Kg} / 100 \mathrm{l}$. Stomach and the liver samples were fixed in $10 \%$ formalin, embedded in paraffin wax, sectioned at $6 \mu \mathrm{m}$ and stained with haematoxylin-eosin. In both stress conditions, the stomach showed important morphological changes in the mucosa layer, such as hypertrophy and atrophy. In the liver, coloration and texture changes were observed as well as haemolisis, inflammation and necrosis. In the water decrease model, the mucosa layer of stomach showed significant morphometric differences $(\mathrm{P}<0.05)$, between different times of stress exposure and/or between different days, in some of the measured parameters. In the high-density model, significant differences in the stomach mucosa showed a sampling-density interaction in 4 parameters and 6 other parameters showed differences with no interaction. Liver showed significant differences in nuclear perimeter between sampling-days. Stress caused by high-density culture proved to be the factor that caused the most serious tissue damage.

\section{KEY WORDS: Stress; Paralabrax maculatofasciatus; Histophatology.}

\section{REFERENCIAS BIBLIOGRÁFICAS}

Anderson, D. P. Immunological indicators: Effects of environmental stress on immune protection and disease outbreaks. Am. Fish. Soc. Symp., 8:38-50, 1990.

Brown, S. B.; Eales, J. G.; Evans, R. E. \& Toshiaki, J. H. Interrenal, thyroidal, and carbohydrate responses of rainbow trout (Salmo gairdneri) to environmental acidification. Can. J. Fish. Aquat. Sci., 41:36-45, 1984.

Cahn, P. H. The pathology of the liver and spleen in naturally stressed Atlantic menhaden. In: The pathology of fishes (ed. Ribelin, E. W. \& Migaki, G.). Madison (WI), The University of Wisconsin Press, 1975.

Cousin, J. C. B. ; Balouet, G. \& Baudin-Laurencin, F. Altérations histologiques observées chez des larves de turbot (Scophthalmus maximus L.) in élevage intensif. Aquaculture, 52:173-89, 1986.

Del Río, O. B. Efecto del estrés térmico sobre los parámetros sanguíneos de la tilapia Orechromis mossambicus (Peters, 1852) (Pices:Cichlidae). Master's thesis, Centro de Investigación Científica y de Educación Superior de Ensenada (CICESE), México, 2004.

Donaldson, E. M. The pituitary-Interrenal axis as an indicator of stress in fish. In: Stress and fish (ed. Pickering, A. D.). London and New York, Academic Press, 1981.

Establier, R.; Gutiérrez, M. \& Arias, A. Acumulación y efectos histopatológicos del mercurio inorgánico y orgánico en la lisa (Mugil auratus Risso). Investigación Pesquera, 42:65-80, 1978.
Estrada, F. E.; Peralta, L. \& Rivas, P. Manual de técnicas histológicas. México D. F. AGT Editor, 1982.

Ferguson, H. W. Systemic pathology of fish. Iowa, Iowa State University Press, 1992.

Fjolstad, M. \& Heyeraas, A. L. Muscular and myocardial degeneration in cultured Atlantic salmon, Salmo salar L., suffering from "Hitra disease". J. Fish Dis., 8:36772,1985 .

Goede, R. W. \& Barton, B. A. Organismic indices and an autopsy-based assessment as indicators of health and condition of fish. Am. Fish. Soc. Symp., 8:93-108, 1990.

Hästein, T. \& Lindstad, T. Diseases in wild and cultured salmon: Possible interactions. Aquaculture, 98:277-88, 1991.

Hernández, M. Comportamiento termoregulador de Poecilia sphenops (Pisces:Poecilidae) aclimatada a temperaturas constantes y fluctuantes. D. Sc. Thesis, Centro de Investigación y de Educación Superior de Ensenada (CICESE), México, 1998.

Hernández, J. R. Efecto de la temperatura crítica máxima y la letal superior sobre el tejido hepático y renal de la sardina de Pacífico, Sardinops sagax (Jenyns, 1842). Master's thesis, Centro de Investigación Científica y de Educación Superior de Ensenada (CICESE), México, 2006.

Hinton, D. E. \& Laurén, D. J. Integrative histopathological approaches to detecting effects of environmental 
stressors on fishes. Am. Fish. Soc. Symp., 8:51-66, 1990.

Jiménez, F.; Galviz, L. \& Segovia, F. Parásitos y enfermedades de la lobina. México, Fondo pesca, Facultad de Ciencias Biológicas UANL, 1986.

Lesel, R. The influence of stress on the microflora of the digestive tract in the rainbow trout, Salmo gairdneri Richardson. International Symposium, "Stress and fish", University of East Anglia, Norwich. In: Stress and fish (ed. Pickering, A. D.). London and New York, Academic Press, 1980.

Olfert, E. D.; Cross, B. M. \& McWilliam, A. A. Manual sobre el cuidado y uso de los animales de experimentación. $2^{\text {a }}$ Ed. Ottawa, Consejo Canadiense de Protección de los animales, 1998.

Mazeaud, M. M.; Mazeaud, F. \& Donaldson, E. M. Primary and secondary effects of stress in fish: some new data with a general review. Trans. Am. Fish Soc., 106:20112, 1977.

Olojo, E. A. A.; Olurin, K. B.; Baka, G. M. \& Olowemimo, A. D. Histopathology of the gill and liver tissues of the Efrican catfish Clarias gariepinus exposed to lead. Afr. J. Biotechnol., 4(1):117-22, 2005.

Peters, G.; Klinger, H. \& Delventhal, H. Stress diagnosis of eels. International Symposium "Stress and fish", University of East Anglia, Norwich. In: Stress and fish (ed. Pickering, A. D.). London and New York, Academic Press, 1980.

Pickering, A. D. Growth and stress in fish production. Aquaculture, 111:51-63, 1993.

Roberts, R. J. Melanin-containing cells of teleost fish and their relation to disease. In: The pathology of fishes (ed. by Ribelin, E. W. \& Migaki, G.). Madison (WI), The University of Wisconsin Press, 1975.

Salam, B. A.; Nesa, B. \& Nessa, Q. Effects of sumithion on the histological changes of spotted murrel, Channa punctatus (Blonch). Pak. J. Biol. Sci., 4(10):1288-90, 2001.

Sindermann, C. J. Disease, diagnosis and control in North American marine aquaculture. Amsterdam, Eisevier Scientific Publishing Company, 1977.

Sindermann, C. J. Principal diseases of marine fish and shellfish. $2^{\mathrm{a}}$ ed. San Diego, Academic Press, 1990.
Tave, D. Selection for stress response to improve disease resistance in Atlantic salmon and Rainbow trout. Aquaculture Magazine, 20:80-4, 1994.

Titu, V. Temperatura as a midifier of fish cardiac contractility. Thesis, University of Joensuu, 2002.

Walling, J. E. \& Van Dén Avyle, M. J. Interactive effects of stocking site salinity and handling stress on survival of Striped bass Fingerlings. Trans. Am. Fish Soc., 124:73645, 1995.

Wedemeyer, G. A. \& McLeay, D. J. Methods for determining the tolerance of fishes to environmental stressors. In: Stress and fish (ed. Pickering, A. D.). London and New York, Academic Press, 1981.

Wolf, K. \& Smith, C. E. Herpesvirus salmonis: pathological changes in parenterally-infected rainbow trout, Salmo gairdneri Richardson, fry. J. Fish Dis., 4:445-57, 1981.

Dirección para correspondencia:

Dra. Patricia Rivas

Laboratorio de Biología de la Reproducción Animal. Facultad de Ciencias

Universidad Nacional Autónoma de México.

Ciudad Universitaria, Circuito Exterior. C.P. 04510.

México, D.F.

MEXICO

Fax: (55) 56224828.

E-mail: prma@fciencias.unam.mx magali@exalumno.unam.mx

Recibido : 06-11-2008

Aceptado: 24-02-2009 
